\section{Clozapine-induced liver injury and pleural effusion}

\author{
Joseph P.M. Kane, Francis A. O’Neill \\ Centre for Public Health, Institute of \\ Clinical Sciences, Queens University \\ Belfast Royal Victoria Hospital, UK
}

\begin{abstract}
Clozapine, whilst associated commonly with a transient and benign increase in liver enzymes, has also been associated with varying presentations of hepatitis in existing case reports. This report describes what we believe to be the first documented case of acute liver injury and pleural effusion associated with clozapine, resolving after cessation of the agent. The case supports existing literature in advocating a high index of suspicion, particularly in the 4-5 weeks following clozapine initiation, when considering nonspecific clinical symptoms and signs.
\end{abstract}

\section{Introduction}

Clozapine is an atypical antipsychotic and a highly effective agent in the management of treatment resistant schizophrenia. ${ }^{1}$ Whilst associated commonly with a transient and benign increase in liver enzymes, ${ }^{2}$ it also lists hepatitis amongst its side effects. Existing case studies report a wide variation in comorbid characteristics and biochemical profiles of such presentations. In this case study we describe a case of clozapine-induced hepatotoxicity with associated pleural effusion.

\section{Case Report}

Ms. A, a 47-year-old woman with a longstanding diagnosis of schizophrenia, was commenced on clozapine therapy having suffered a relapse characterized by an escalation in auditory hallucinations, persecutory thought, behavioral disturbance and negative symptoms; treatment with both atypical (olanzapine, risperidone) and typical (haloperidol) antipsychotic agents had been unsuccessful. No significant medical disorders were present in the patient's past medical history or family history, nor was there a history of drug allergies. There was no history of alcohol or illicit drug abuse. She described a smoking history of 30 pack years. Baseline electrocardiograph and blood tests, including liver function tests (LFTs), full blood count, fasting lipid profile and glucose, were all unremarkable.

Daily clozapine dose was titrated to $400 \mathrm{mg}$ over a three week period with mild sedation alone noted as a side effect. A tangible improvement in MsA's mental state was noted over this period, with improvement in both positive and negative symptoms.

Thirty days following clozapine prescription, the patient developed lethargy and pyrexia, cough and left basal crepitations. Blood investigations revealed an elevated white cell count (WCC $14.4 \times 109 / \mathrm{L}$ ), characterized by eosinophilia $\left(2.39 \times 10^{9} / \mathrm{L}\right)$, and elevated $\mathrm{C}$ reactive protein (CRP). LFTs returned deranged values of bilirubin $23 \mathrm{umol} / \mathrm{L}$ (normal range 3-18 umol/L), alkaline phosphatase (ALP) 220 U/L (n.r. 35-120 U/L), aspartate transaminase (AST) 338 U/L (n.r. 4-32), gamma-glutamyl transpeptidase (GGT) $96 \mathrm{U} / \mathrm{L}$ (n.r. 12-58 U/L), and alanine transaminase (ALT) 894 U/L (n.r. 10-35 U/L).

Symptoms persisted for several days, during which chest X-ray indicated the presence of a small left basal effusion. Although initially diagnosed as a lower respiratory chest infection and treated with Co-amoxiclav, clarithromycin, and subsequently piperacillin/ tazobactam, all antibiotic agents were discontinued seven days after onset of symptoms when concern arose that the agents were both ineffective and contributing to LFT derangement.

Abdominal ultrasound was normal, while copper and caeruloplasmin levels were within reference ranges. Hepatitis A,B,C and E serology tests were negative, as were CMV and EBV serology. Levels of serum immunoglobulins and autoantibodies [(antinuclear antibodies (ANA), anti-smooth muscle antibodies (ASMA), anti-liver-kidney microsome-1 antibodies (ALKM-1) and anti-liver cytosol antibody-1 (ALC-1)] were within reference ranges and serum paracetamol levels were measured at less than $0.1 \mathrm{mmo} / \mathrm{L}$. Whilst magnetic resonance imaging studies and pleural effusion aspiration were scheduled, these were precluded by the patient withholding her consent to undergo these investigations.

The patient deteriorated clinically over the following days, developing jaundice and persisting lethargy. Liver enzymes reached peak values of bilirubin $86 \mathrm{umol} / \mathrm{L}$, ALP $406 \mathrm{U} / \mathrm{L}$, AST $569 \mathrm{U} / \mathrm{L}, \mathrm{GGT} 173 \mathrm{U} / \mathrm{L}$, and ALT $707 \mathrm{U} / \mathrm{L}$ before clozapine was discontinued, 40 days following initial prescription. Liver enzymes reduced markedly from two days after cessation, returning to within normal reference ranges within six weeks. All evidence of pleural effusion had disappeared from chest x-ray within two days of clozapine cessation.
Correspondence: Joseph P.M. Kane, Centre for Public Health, Institute of Clinical Sciences, Queens University Belfast Royal Victoria Hospital, Grosvenor Road, Belfast BT12 6BA, UK. Tel.: +44.289.063.2580

E-mail: jkane11@qub.ac.uk

Key words: paranoid schizophrenia, clozapine, adverse effects.

Contributions: the authors contributed equally.

Conflict of interests: the authors declare no potential conflict of interests.

Received for publication: 31 March 2014.

Revision received: 11 May 2014.

Accepted for publication: 20 May 2014.

This work is licensed under a Creative Commons Attribution NonCommercial 3.0 License (CC BYNC 3.0).

(O)Copyright J.P.M. Kane and F.A. O'Neill, 2014

Licensee PAGEPress, Italy

Mental Illness 2014; 6:5403

doi:10.4081/mi.2014.5403

\section{Discussion and Conclusions}

We report a case of drug-induced liver injury with pleural effusion as a side effect of clozapine therapy. Although transient LFT elevation is well recognized in the period following clozapine prescription, ${ }^{2}$ liver injury is a rare side effect of clozapine therapy; to date only five cases of mortality secondary to hepatic failure associated with clozapine therapy have been reported in the United Kingdom. ${ }^{3}$ Current guidelines, however, advocate routine LFT monitoring only at baseline and every six months following prescription.

Other cases of drug-induced liver injury have been described in the existing literature, ${ }^{4-8}$ though none with associated pleural effusion. Whilst Thompson et al. ${ }^{9}$ describe a case involving hepatitis and bilateral pleural effusion, together with hematuria and proteinuria, insult was of an obstructive, rather than hepatocellular mechanism, and symptoms occurred far earlier in the course of treatment (18 days).$^{9}$

Two distinct mechanisms for such reactions arise from the documented cases. Ms. A's eosinophilia and elevated inflammatory markers suggest an etiology more closely aligned to the immunoallergic reaction observed in approximately half of the cases documented, than with the idiosyncratic responses described in the remainder. Our case is comparable with other reported cases of liver toxi- 
city in onset of symptoms (4-5 weeks following clozapine initiation), clozapine dose (300-500 $\mathrm{mg}$ daily) and duration before normalization of laboratory results (4-6 weeks), although the LFT figures reported in Ms. A's case surpass those described in existing literature. It should be noted that in many of these cases, clinical symptoms were non-specific, as in Ms. A's case, or absent altogether.

The authors fully acknowledge that the patient's pleural effusion may have occurred due to other etiologies, an assertion that may have been supported by investigations declined by Ms. A. We do, however, believe that the close correlation of clinical symptoms with laboratory tests and the rapid resolution of effusion in the absence of antibiotic therapy support an association with hepatotoxicity. It is also noted that clozapine-induced pleural effusion is not unprecedented in the literature.

Whilst both the unsuccessful and successful re-challenges with clozapine therapy following adverse reactions are documented in existing reports, $6,7,10$ re-challenge was considered inappropriate in Ms. A's case, given the extent of LFT derangement and clinical symptoms.

This case supports existing literature in advocating a high index of suspicion, particularly in the 4-5 weeks following clozapine initiation, when considering clinical symptoms and signs commonly associated with other pathologies. Whilst the documented prevalence of transient LFT elevation should urge caution in the premature cessation of clozapine therapy, clinicians should maintain a low threshold for monitoring such parameters following the emergence of innocuous symptoms and signs.

\section{References}

1. Kane J, Honigfeld G, Singer J, Meltzer H. Clozapine for the treatment-resistant schizophrenic. A double-blind comparison with chlorpromazine. Arch Gen Psychiatry 1988;45:789-96.

2. Hummer M, Kurz M, Kurzthaler I, et al. Hepatotoxicity of clozapine. J Clin Psychopharmacol 1997;17:314-7.

3. Medicines and Healthcare products Regulatory Agency (MHRA). Drug Analysis Prints (DAPs). Medicines and Healthcare products Regulatory Agency. Available from: http://www.mhra.gov.uk/ Safetyinformation/Howwemonitorthesafetyofproduct s/Medicines/TheYellowCardScheme/Yello wCarddata/Druganalysisprints/index.htm\# retainDisplay. Accessed: 16th March 2014.

4. Kellner M, Wiedemann K, Krieg JC, Berg
PA. Toxic hepatitis by clozapine treatment. Am J Psychiat. 1993;150:985-6.

5. Thatcher GW, Cates M, Bair B. Clozapineinduced toxic hepatitis. Am J Psychiat 1995;152:296-7.

6. Markowitz JS, Grinberg R, Jackson C. Marked liver enzyme elevations with clozapine. J Clin Psychopharmacol 1997;17:70-1.

7. Keane S, Lane A, Larkin T, Clarke M. Management of clozapine-related hepatotoxicity. J Clin Psychopharmacol 2009;29: 606-7.

8. Macfarlane B, Davies S, Mannan K, et al. Fatal acute fulminant liver failure due to clozapine: a case report and review of clozapine-induced hepatotoxicity. Gastroenterology 1997;112:1707-9.

9. Thompson J, Chengappa K. Hepatitis, hyperglycemia, pleural effusion, eosinophilia, hematuria and proteinuria occurring early in clozapine treatment. Int Clin Psychopharm 1998;13:95-8.

10. Eggert AE, Crismon ML, Dorson PG, Taylor RL. Clozapine rechallenge after marked liver enzyme elevation. J Clin Psychopharmacol 1994;14:425-6. 